INTERNATIONAL DESIGN CONFERENCE - DESIGN 2018

https://doi.org/10.21278/idc.2018.0200

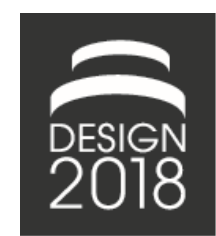

\title{
DESIGN EXPLORATION OF BIOMORPHIC FREEFORM UNIT CELLS FOR ADDITIVELY MANUFACTURED LATTICE STRUCTURES UNDER COMPRESSIVE LOADS
}

\author{
A. Thallemer, A. Kostadinov, A. Fam and A. Teo
}

\begin{abstract}
Additive manufacturing methods facilitate the production of complex cellular materials. Commonly, their designs are based on primitive solids using linear struts without curvature continuous joints which results in notch stress. This study presents cellular material units with biomorphic features. The employed method allows us to design lattices with geometrical optimization and varying lattice morphology. The compression test results show that this method allows us to achieve a spectrum of mechanical properties for improving existing 3D printed lattice materials.
\end{abstract}

Keywords: biologically inspired design, lattice structures, additive manufacturing, lightweight design

\section{Introduction}

Cellular materials like foams and lattices have major advantages over solid components as structural elements by saving material and weight, and thus increasing the economic aspects of production and use (Zhang et al., 2015; Li et al., 2017). Non-stochastic lattices represent a subset of cellular materials (Gibson and Ashby, 1988; Sun and, Li 2018) with long range periodicity and clearly defined boundaries (Zok et al., 2016). These distinctive features distinguish them from porous material, making them superior to stochastic cellular materials like foams when it comes to load bearing applications (Zok et al., 2016; Darabi, 2017; Sun and Li, 2018).

The Eiffel tower (1889), known for its high strength to lightweight ratio, represents one of the earliest successes of lattice design applied to a megastructure (Lakes, 1993; Sundaram and Ananthasuresh, 2009). Today, microfabrication technologies like Additive Manufacturing (AM) allow lattice structures with complex morphological designs to be accurately produced at microscopic (100 nm to $100 \mu \mathrm{m})$ spatial length scales (Vaezi et al., 2013). Additionally, lattice materials facilitate stiffness-modulation (Bottlang et al., 2010), which is highly relevant for engineering bone implants to prevent stress shielding effects (Cronskär, 2011). Pushing these quasi-compliant structures further through rationally-designed elastic instability, lattices can be useful for energy absorption and shock dampening (Sanami et al., 2014; Qiao and Chen, 2015; Sun and Li, 2018). Apart from functional optimization, these "soft" structures also provide key functionalities in areas like soft robotics and soft electronics (Kolken and Zadpoor, 2017). Therefore, understanding deformation mechanics in lattice materials is highly relevant when it comes to their mechanical applications.

One way to identify deformation mechanics in lattice structure is to differentiate between stretch- and bend-dominated architectures. It has been established that the former exhibit greater stiffness and strength, making them suitable for rigid structures (Deshpande et al., 2001a), while the latter are mainly 
used as compliant structures (Saunders, 2017). The deformation mode is primarily predicted by nodal connectivity (Deshpande et al., 2001a; Fleck, 2004) and Maxwell's Criterion for determining structural rigidity (Fleck, 2004). This well-established approach, however, does not fully consider the possibilities which lie within a lattice's design.

First, these methods for predicting the mechanical behaviour neglect the node's geometry. Consequently, conventional truss-based designs in literature (Ahmadi et al., 2015; Liu et al., 2018) often lack curvature transitions between intersecting trusses. This feature decreasesresults to unoptimized nodes that decrease the potential strength and durability of the structure, because such notch stressed nodes might often cause aresult in premature failure (Helou and Kara, 2017). Secondly, the aforementioned predictive methods have mostly been applied to conventional truss-based lattices which were constructed from primitive geometries utilising linear struts (Deshpande et al., 2001b), and therefore the methods might not be sufficient to predict the deformation mode of non-linear strut (NLS) lattices. Thirdly, in bend-dominated architectures, unless the large strain or deformation in compliant structures is achieved by elastic instability within a critical value of applied strain (Bertoldi et al., 2010), the bending of linear struts around a rigidjointed node is prone to uncontrolled buckling which can lead to undesired plastic deformation.

Together, these observations create an opportunity for further exploration and understanding of lattices involving morphologies with NLS and well resolved node geometries. Structures which offer those combined characteristics will be hereon referred to as "freeform lattice design" (FLD) structures. Since this paper examines the freeform lattice's potential in a dynamic range of mechanical applications, we will focus on strut-based (both linear and non-linear) lattice designs and exclude triply-periodic minimal surfaces (TPMS) because a surface is more prone to buckling than a slender strut of similar mass (Zok et al., 2016). By subjecting these freeform lattices to compressive loading, our goal is to discover designs with desirable mechanical properties and a potential to serve well in both static and dynamic loading conditions.

\section{Design heuristics of the cell unit}

\subsection{Design exploration process}

In literature, we observed that the common method to designing an inherently three-dimensional unit cells involves space-filling geometries, from which either the boundary frame's edges and vertices are directly traced, or whose volume within the boundary space is filled with other geometric primitives (Helou and Kara, 2017). Such designs are conventionally generated using primitive solids like spheres, cylinders, and prisms. However, this method of assembling by analogy is sub-optimal, as the resulting unit cell is, as aforementioned, prone to notch stress and buckling. Therefore, it is important to explore novel cell types by diverging from the common method of creating a unit cell. A radical approach for a new design would be through the implementation of computer-aided design (CAD) exploration. So-called generative design tools are capable of offering shapes for well and marginally defined problems. Apart from creating arbitrary shapes for aesthetics purposes (McCormack et al., 2005), generative design tools can be used for structural applications where the constraints such as applied forces, materials, and manufacturing methods are known and well defined (Shea et al., 2005). These generative solutions however, are only applicable for exploring individual component geometries that already exist in CAD libraries (Wettergreen et al., 2005). When it comes to creating distinct design variants for periodic unit cells which add up to a distinct mechanical behaviour, the available software applications do not offer any design exploration. To explore a variety of unit cell types, we therefore asked undergraduate industrial design students to develop unit cells from which we anticipated two major advantages. Firstly, we expected the students to be unbiased by crystallographicbased and geometry-based designs for the unit cell geometry. Secondly, knowing that the students are familiar with freeform modelling, we were expecting to obtain curvature continuous geometries. This way, we would achieve two goals simultaneously: node optimization and alternative strut geometry.

\subsection{Design brief and design constraints}

The students were asked to create inherently three-dimensional unit cells which can be tiled in three perpendicular directions (X, Y, and Z) resulting in a cubic or cuboid array configuration (Table 1). The configuration mode was considered the design space which provided the area of topological and geometrical design exploration. To guarantee an unbiased approach towards the structural design, there 
were no indications for a functional application. This intentional lack of a clear purpose was expected to induce divergent results, which would provide a greater variety within the sample group. It was demanded, however, that the students had to construct the surfaces using hyperbolic geometry in order to simultaneously achieve the required surface optimization (Liu et al., 2018). Having those geometric constraints, many of the students derived their designs from the biological world, in a same way a biomorphic ornament is derived, i.e. stimulus and abstraction. This implies, that the students did not analyse the structures, which are known for the mechanical properties. Instead they used only visual impressions as a graphical guidance for their cell unit architecture.

Table 1. Lattices' stacking and array configurations

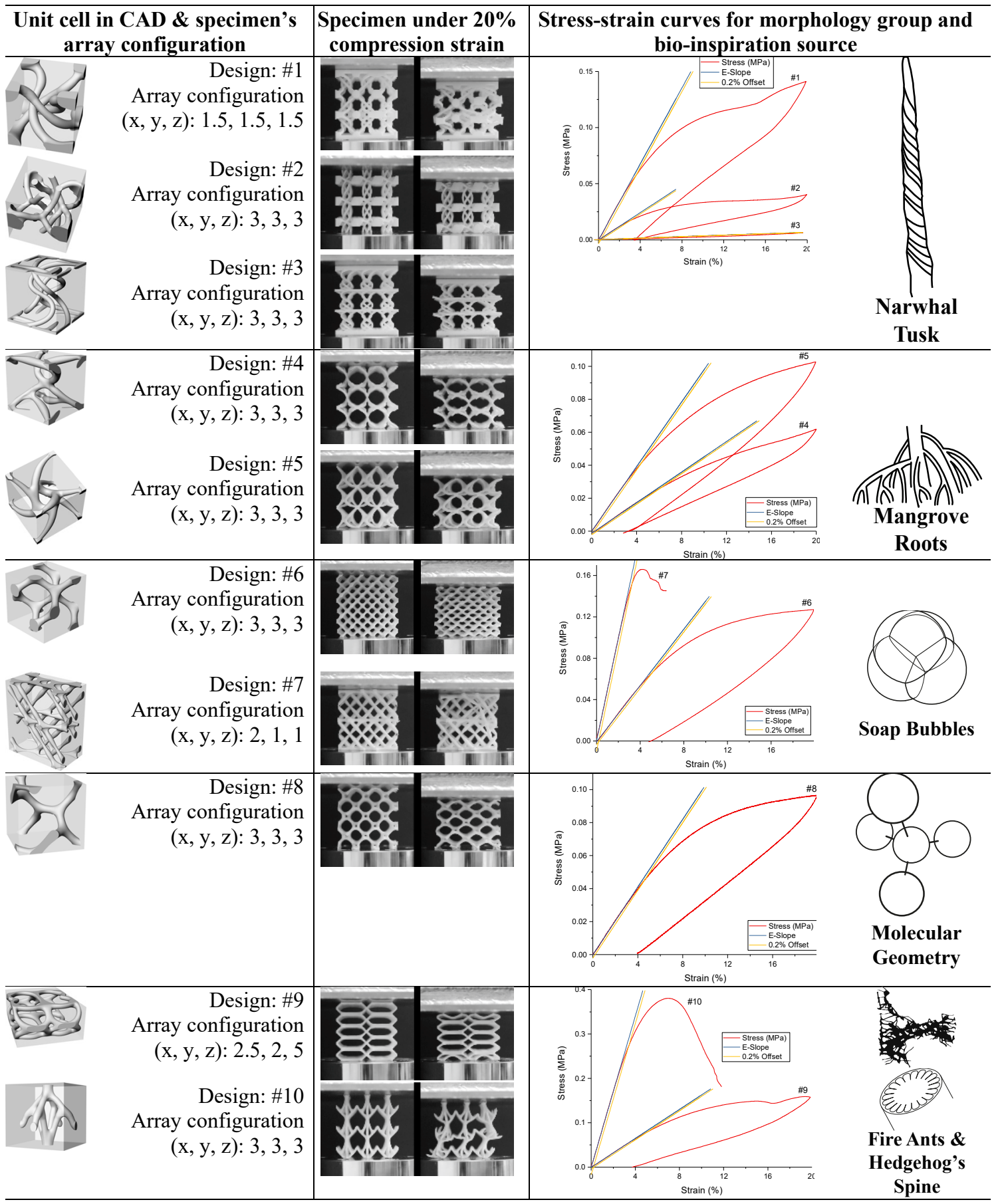




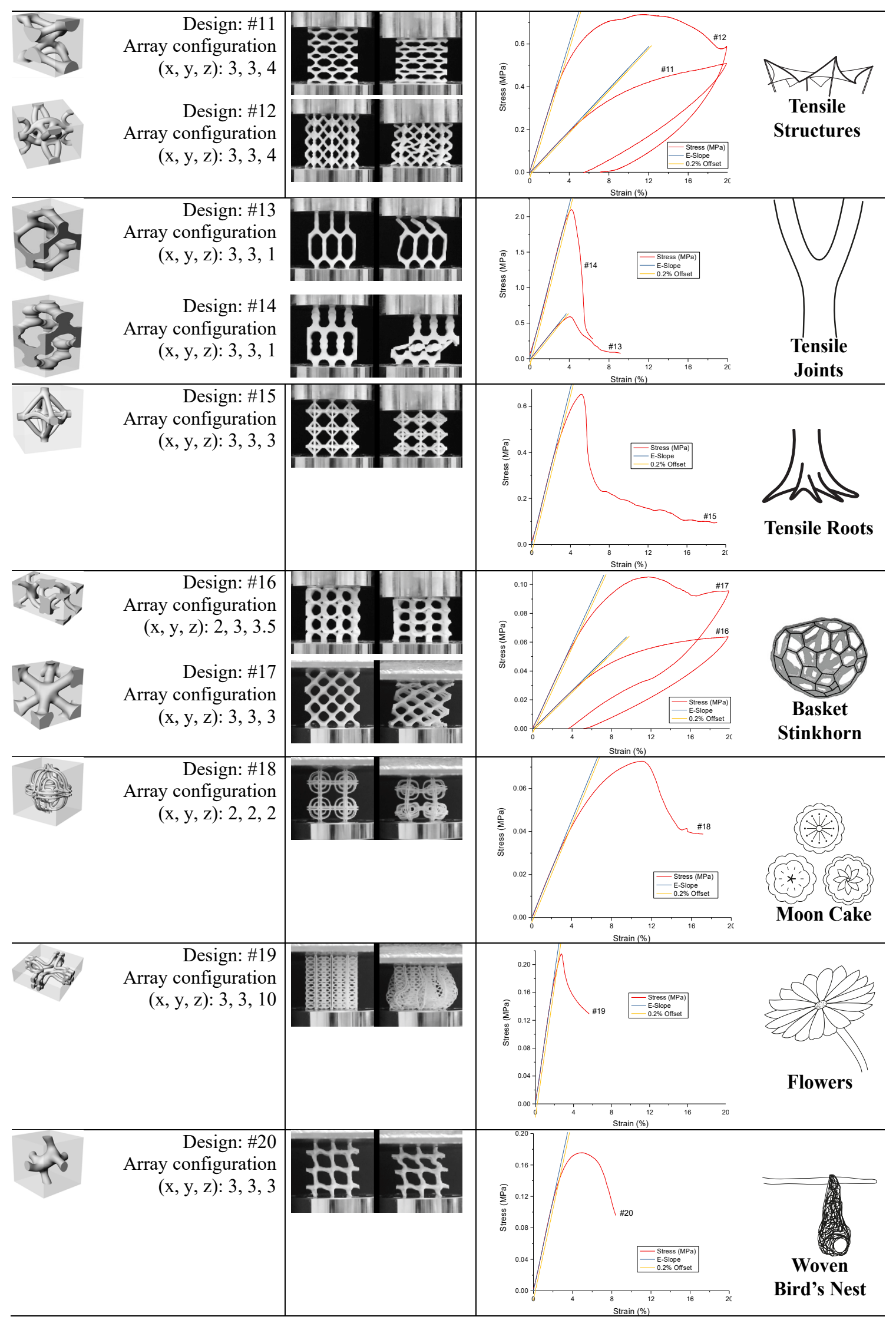




\section{Methods}

\subsection{Experiment and specimen design}

The lattices were manufactured through the Selective Laser Sintering (SLS) powder bed method using PA2200; the details regarding the specimen fabrication will be discussed in Section 4. A compression test was performed with the goal to uncover the potential of each FLD for further design development prior to a contextualized design refinement toward specific applications. Since there was not a contextualized evaluation criterion, each design was expected to exhibit potential in vastly different application areas. The challenging aspect of the testing was to ensure a valid basis for comparative evaluation among the different designs,

Compressive stress is defined as 'the compressive load per unit area of minimum original cross section within the gage boundaries, carried by the test specimen at any given moment, expressed in force per unit area' (ASTM, 2008). Conventionally, the minimum cross section area of the specimen is used for the calculation of compressive stress values. However, in the context of this experiment, each FLD specimen has a different minimum cross section area since this parameter (i.e. physical dimension) is primarily determined by the specific proportion of volume in the hyperbolic node and thickness of each connected struts as determined by the designer. The minimum cross section area was hence considered an intrinsic design property of the FLD. Other intrinsic design properties included parameters such as curvature continuity at the nodes, and stacking mode of the unit cells which determines lattice density within a given volume. These intrinsic design properties form the independent variable, while the dependent variable includes the unique deformation mechanism and macroscale mechanical properties.

Having both cubic and cuboid unit cells, we had to translate the designs into specimens without distortion while maintaining a similar bounding volume, referred to as "bounding box". The specimens were standardised to $50 \mathrm{~mm}$ Z-height to ensure $20 \%$ strain was consistently applied via uniaxial compression. Majority of the designs fit exactly within the $50 \mathrm{~mm}$ x $50 \mathrm{~mm} X-Y$ plane, while some of the cuboid unit cells occupied a smaller X-Y plane. The specimen's cuboid form is in accordance with similar studies (Wang et al., 2016; Qin et al., 2017). However, it is worthy to note that in those papers, the respective experiments involved only one type of lattice topology design. In our compression test, however, the 20 lattice designs are all different, therefore, the independent variable in this experiment is the freeform lattice design, which is defined by its intrinsic design properties (e.g. physical dimensions of struts and nodes). The specimen's minimum cross section area did not serve for the calculation of compressive stress values, instead, the cross-section area of the bounding box's X-Y plane was used for stress calculations.

\subsection{Specimen fabrication}

Test specimens with macro-manufacturing $(>0.5 \mathrm{~mm}$ ) (Dow and Scattergood, 2003) length scales of $50 \mathrm{~mm} \times 50 \mathrm{~mm} \times 50 \mathrm{~mm}$ bounding box volume were prepared using Selective Laser Sintering (SLS) technology, a powder bed based additive manufacturing technology employed by EOS FORMIGA P110 machine. The additive material used was Polyamide (PA) 2200, a derivative of Nylon 12 (Anon, n.d.), which has a layer thickness of $0.06 \mathrm{~mm}$ (Anon, n.d.). While the SLS method allows for detailed features in the surface transitions of each lattice to be successfully fabricated, there will still be microscale imperfections and anisotropy as observed in all additively manufactured products.

Since the study focuses on macroscale mechanical properties due to differences in morphology design, only one specimen was prepared for each design. It is recognised that microscale imperfections would influence the observed macroscopic properties. For designs that features geometric anisotropy, the specimen's orientation in printing complies with the Z-direction as intended by the lattice's designer.

\subsection{Test protocols and instruments}

The absence of a specified application means that a standardized failure criterion, i.e. Ultimate Failure, which would be required for calculating Modulus of Toughness, cannot be defined. Additionally, each lattice design can have a different deformation mechanism and hence a different failure mode. 
Therefore, a pre-test was performed on two solid PA2200 $50 \mathrm{~mm} \times 50 \mathrm{~mm} \times 50 \mathrm{~mm}$ cubes as a benchmark, and an average between the two replicates recorded $0.2 \%$ offset yield point at $1.005 \%$ strain. Given that PA2200 is a derivative of PA12 which has elastic modulus values between 4.28 to 5.31 GPa (Anon, n.d.), the compressive yield point of typical PA12 is about $2.4 \%$ strain. These findings implied that at $20 \%$ strain, the compression test would be able to capture both the elastic and plastic behaviour.

Therefore, the test profile was set to return the crosshead at the same rate upon reaching $20 \%$ strain to measure the extent of permanent deformation by calculating the percentage of energy returned during unloading. The test was truncated for specimens that underwent drastic failure mode before $20 \%$ strain was reached. The drastic failure mode was characterized by stress-strain behaviour where one or several broken struts or complete buckling of unit cells resulted to a phenomenon that resembles a brittle failure or ductile rupture - the two types of ultimate failure in brittle and ductile materials respectively. Gradual ductile failure was not considered as a failure criterion to stop the test, as ductile failure could be a beneficial design characteristic that prevents catastrophic structural failures.

The compression test was standardized with a crosshead speed of $1.6 \mathrm{~mm} / \mathrm{min}$ in both the elastic and plastic region, which was within the strain rate recommended by the ASTM D695 (ASTM, 2008). Two types of test machines were eventually used. Specimens were first tested with the Instron's digital 5543 Universal Testing Machine for its higher sensitivity load cell, with a preload of 0.5 to $1 \mathrm{~N}$. Specimens with peak load exceeding $1 \mathrm{kN}$ had new replicates tested with MTS Corporation's hydraulic Bionix II 858 capable of producing $10 \mathrm{kN}$ crushing force, with a preload of 2 to $4 \mathrm{~N}$. The preloads were applied to ensure full contact between specimen and crosshead. The sampling frequency was standardized at 5 $\mathrm{Hz}$ for both machines. The specimens were directly compressed by two parallel crossheads with metallic jig attachments featuring large and flat surface area far exceeding the specimens' bounding box. Specimens were not adhesively bonded to the jig so that the different deformation mechanisms could be observed. For instance, the displacement of struts redistributes stress as strain increases. These observations were captured by 50fps, $720 \mathrm{p}$ resolution video recordings at orthogonal and perspective views of the specimen. Still images of the pre-loaded and $20 \%$ strained specimens are shown in the following section.

\subsection{Pretext for the mechanical evaluation}

In the evaluation of cellular structures for mechanical applications, there are several perspectives to consider (Sun and Li, 2018). The primary of them is the definition of the general loading condition: static or dynamic. In both conditions, infinitesimal strain represents the elastic deformation of materials where the undeformed and deformed state can be assumed identical. Lattices with stretch-dominated architecture octet truss is a geometry-based design which are well suited for those applications. Having high strength and stiffness, as well as structural rigidity (Deshpande et al., 2001b), those structures permit higher loads below the yield point without significant structural deformation and subsequent elastic recovery. A second category of dynamic loading is described by the finite strain theory, where a material experiences large strain and/or rotation, causing undeformed and deformed state of the continuum to be significantly different. It is generally observed in literature, that bend-dominated architectures are preferred for such conditions. They are used in applications like energy absorption and shock dampening, which require soft, compliant structures since they provide a large crumping zone upon compressive loading (Ashby, 2005; Fleck, 2004).

However, a generally identified trade-off is that bend-dominated lattices tend to have significantly lower strength and stiffness than their stretch-dominated counterparts (Deshpande et al., 2001a; Fleck 2004). It is also noted that in large enough strain levels, large compressive deformation experienced by the bend-dominated lattice beyond the elastic limit would involve plastically-deforming struts around a rigid-jointed node. Consequently, permanent deformation experienced by the bent struts will eventually reduce the efficacy and durability of the lattice material in its application.

While the trade-offs in design implementation between the stretch- versus bend-dominated architecture model is well understood, we would argue that this model does not holistically consider the above mechanical aspects of "static or dynamic" and "infinitesimal or finite" strain conditions. It is therefore not inconceivable to imagine a novel lattice design with mechanical properties optimized for a specific application. A recently 
popularized example of such a lattice material belongs to a class of mechanical metamaterials exhibiting Negative Poisson Ratio (NPR), also known as auxetics (Carneiro et al., 2013; Kolken and Zadpoor, 2017). They possess rationally-designed structural instability, along with high stiffness, which makes them ideal for attenuating high velocity impacts. Interestingly, three-dimensional auxetics are not primarily classified through the aforementioned stretch- and bend-dominated architecture model. Instead, they are more commonly categorized into re-entrant or chiral designs, where each category has a different deformation mechanism e.g. rib hinging and rotating to achieve elastic instability (Mir et al., 2014).

Considering these observations, we think that going beyond auxetic lattices that are favoured for compliant applications and conventional geometry-based designs that are favoured for structural stability, our research interest is to explore a wider range of lattice morphologies and hence different types of mechanical behaviour achievable through FLD. A well-tuned proportion between a hyperbolic node and its connecting NLS could bring about a unique deformation mechanism due to the kinematics within each NLS or around the node.

\section{Discussion of results}

\subsection{Method for data analysis and modelling}

When examining lattice materials in literature and commercial contexts, data concerning lattice materials generally display their yield strength, elastic modulus (Mod-E) and sometimes fracture toughness (Anon, n.d.; Ashby, 2005; Vigliotti and Pasini, 2013; Ahmadi et al., 2015). These perspectives favour the evaluation of lattice materials in applications requiring structural stability where high strength and stiffness is prioritised. Differentiating lattice designs based on predicted bend versus stretch-dominated architectures similarly does not provide sufficient information for identifying the design's potential application, since elastic strength values, as well as its elastic-plastic compressive behaviour plays a significant role.

The three monographs (Figures 1, 2 and 3) visualise individual stress strain properties of each design in the ordinate and their respective volume fraction (\%) in the abscissa. Cross-referencing trends across the three individual monographs allows us to observe the effects of specific lattice design parameters on the macroscopic stress-strain properties of lattice structures. By specifically evaluating individual stress strain properties of each design against the overall trends, as well as the known assumptions of compressive stress-strain properties in conventional materials, we can identify designs with anomalous properties, which can be positive (i.e. valuable for a specific application) or negative (i.e. a blend of relatively weak stress-strain properties that does not justify for further optimisation in any specific application). Designs with positive anomalous properties can be recommended for refinement in further design optimisation processes. The identified design with said positive anomalous properties can also serve as a reference for generating more valuable design variants which can then be tested against the original. For comparatively evaluating each design, the respective stress-strain properties are ranked by their mathematical value in descending order, (i.e. for Elastic Modulus, a Rank 1 has higher MPa values than a Rank 2). Since there is not a defined application, the rankings do not represent a design's efficacy but serve only for comparative evaluation of stress-strain properties among the 20 freeform lattice designs.

\subsection{Significance of data modelling}

With the $20 \%$ strain compression data of all 20 freeform lattice designs, we visualised strain at offset yield point (\%) and yield strength (MPa) data as ordinate values in separate plots as opposed to using Modulus of Resilience (i.e. the area under curve for the elastic region) to represent both of these StressStrain data in a single plot is because Modulus of Resilience subsume both of these stress-strain data into a single mathematical value and does not visualize the extent of structural deformation when a certain level of stress is experienced by the specimen. This means that two designs can have similar Modulus of Resilience values even if one has higher yield strength and much lower elastic strain, while the other has the opposite properties. Therefore, visualizing them in different plots would allow the comparison of stress-strain properties as separate parameters that are crucial to design. 


\subsection{Observational insights}

We expected to see whether within the same lattice design, an increasing volume fraction (thicker struts and more voluminous nodes) would correlate with increased yield strength and Mod-E values. This is coherent with the overall trend in Figure 1 which suggests that higher material volume generally leads to high offset yield strength and Mod-E values. However, designs with positive anomalies were also noted in Figure 1, which demonstrates that volume fraction is not the main determinant for higher strength and stiffness. With the statistical mean value for offset yield strength as $0.266 \mathrm{MPa}$, designs \#10, \#13 and \#15 were ranked $2 \mathrm{nd}$, 3rd and 6th respectively. Their yield strength values lie above the average despite having volume fraction below the statistical mean at $9.96 \%$. This implies that strength and stiffness properties are greatly determined by unit cell design and the freeform lattice's morphology. Designs \#10, \#13 and \#15 are hence high potential candidates for high strength and lightweight applications.

Similarly, the overall trend in Figure 2 also suggests that volume fraction had little correlation with the observed strain at offset yield point (\%). This means that the extent of structural deflection is determined by the freeform lattice's morphology, which in turn dictates the type of deformation mechanism. In Figure 3, only the 12 designs that have a return curve after $20 \%$ compressive strain were visualized. When comparing Figure 2 with Figure 3, the overall trend suggests that designs capable of higher yield strain generally have a higher percentage of energy recovered, which implies a lower extent of permanent deformation. This can be accounted by the fact that higher yield strain relates to a greater proportion of area under curve for the elastic than plastic region under $20 \%$ strain condition.

Another positive anomaly was noted in Figure 2 Design \#16 ranked 3rd in yield strain (\%) which implies a relatively high extent of elastic deformation when strained below the yield point. A return curve (Figure 3) was also recorded as no drastic failure was observed under 20\% strain. Despite the structural flexibility, \#16 also ranked 5th in yield strength with $0.334 \mathrm{MPa}$, which is above the statistical mean. The balance between relatively higher strength and extent of elastic deformation suggests that \#16 does not conform to conventional material properties and could be further optimised for potential in high strength, compliant structures which require large strain.

However, it was also observed that most of the freeform lattices exhibit compressive stress-strain properties typical for conventional materials. For instance, high Mod-E value implies high stiffness, and brittleness hence becomes an expected consequence. Designs \#10,\#13 and \#15 identified for their relatively high strength and lightweight experienced drastic failure immediately after the yield point, which is characteristic of brittle materials. The converse is true, where lower stiffness correlates with ductility. Designs with lower yield strength (i.e. the 14 designs ranked 7 th to 20th with offset yield point below $0.266 \mathrm{MPa}$, the statistical mean) tend to have higher ductility. Of these 14 designs, 12 of them did not exhibit failure mode even up till $20 \%$ compressive strain and a return curve was recorded. These 12 designs also exhibited higher strength as strain increases, a phenomenon that is similar to strain hardening. Such a mechanical process is commonly employed to relatively strong and malleable materials like metals (e.g. low carbon steel, pure copper and aluminium) which allows them to undergo work hardening, as opposed to high strength crystalline solids like diamond being brittle despite its impressive mechanical strength. In Figure 1 all designs exhibited strain at offset yield point (\%) above 1.005\% yield strain (as benchmarked by the 2 solid PA2200 $50 \mathrm{~mm} \times 50 \mathrm{~mm} \times 50 \mathrm{~mm}$ cubes), which shows more significant structural compliance as expected of lattice materials.

In terms of deformation mechanism, designs with characteristic ductility were generally observed to have either a homogenous deformation mode throughout the lattice or slight barrelling at higher strain. This is as opposed to simultaneous collapsing of top and bottom layers or layer-by-layer buckling in the lattice for designs with strength and stiffness above the statistical mean. 


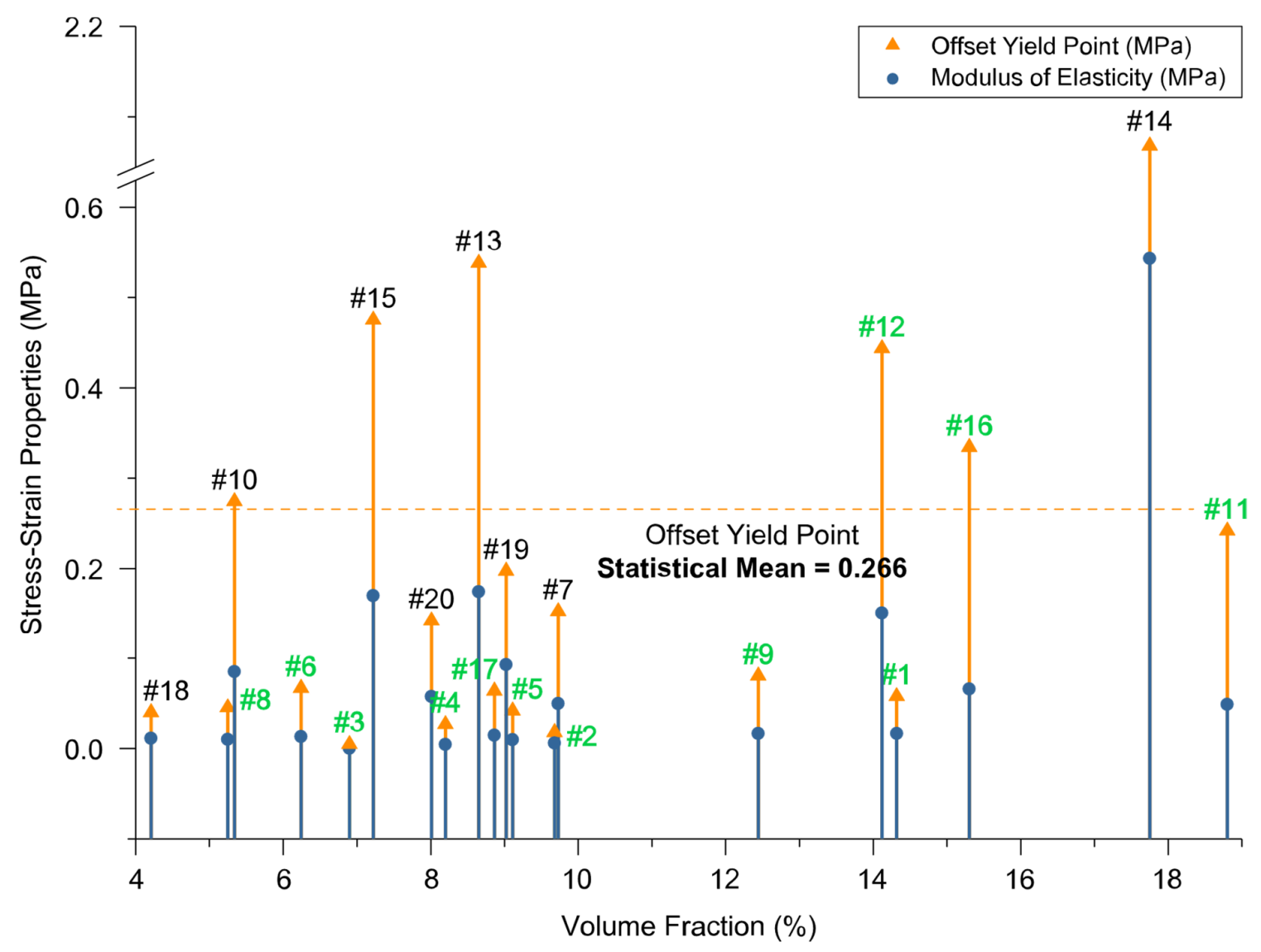

Figure 1. Overview of lattices' offset yield points and moduli of elasticity, the 12 green labelled designs indicate that a return curve was recorded

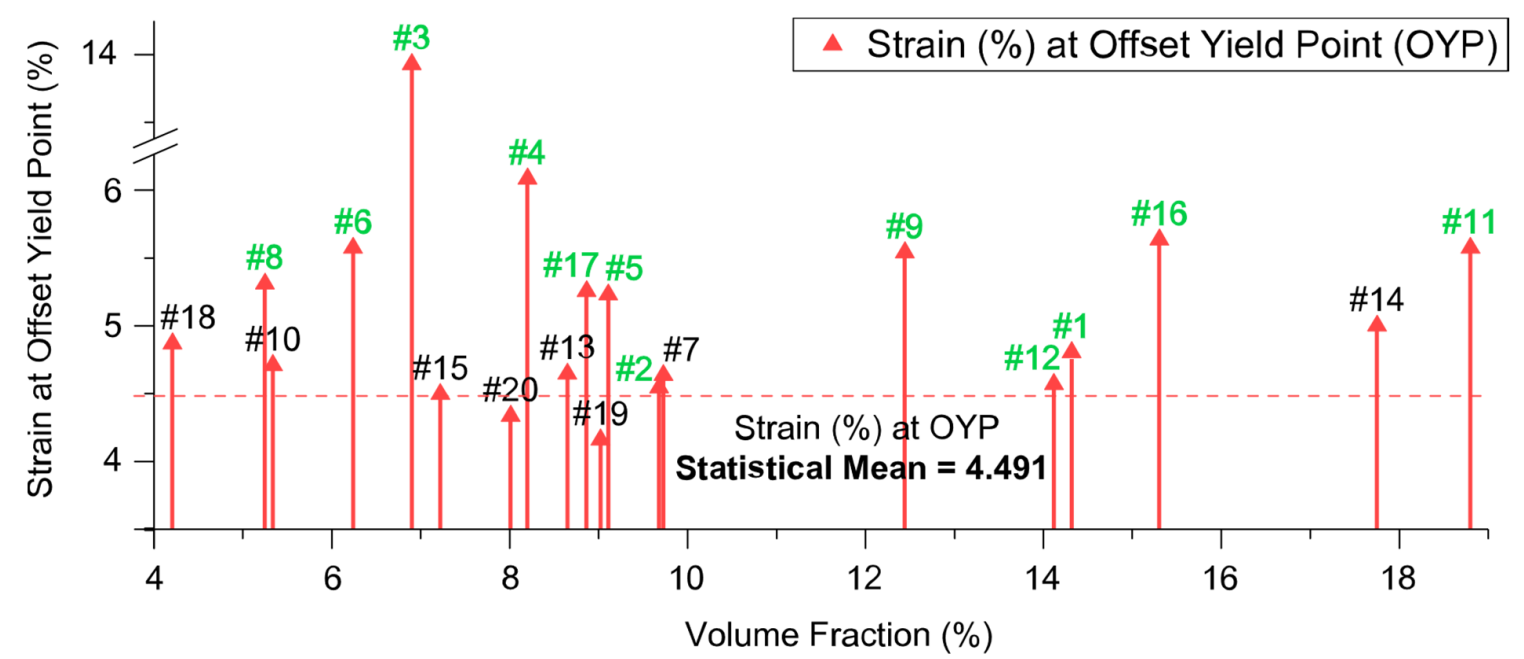

Figure 2. Overview of lattices' strain at offset yield point, the 12 green labelled designs indicate that a return curve was recorded 


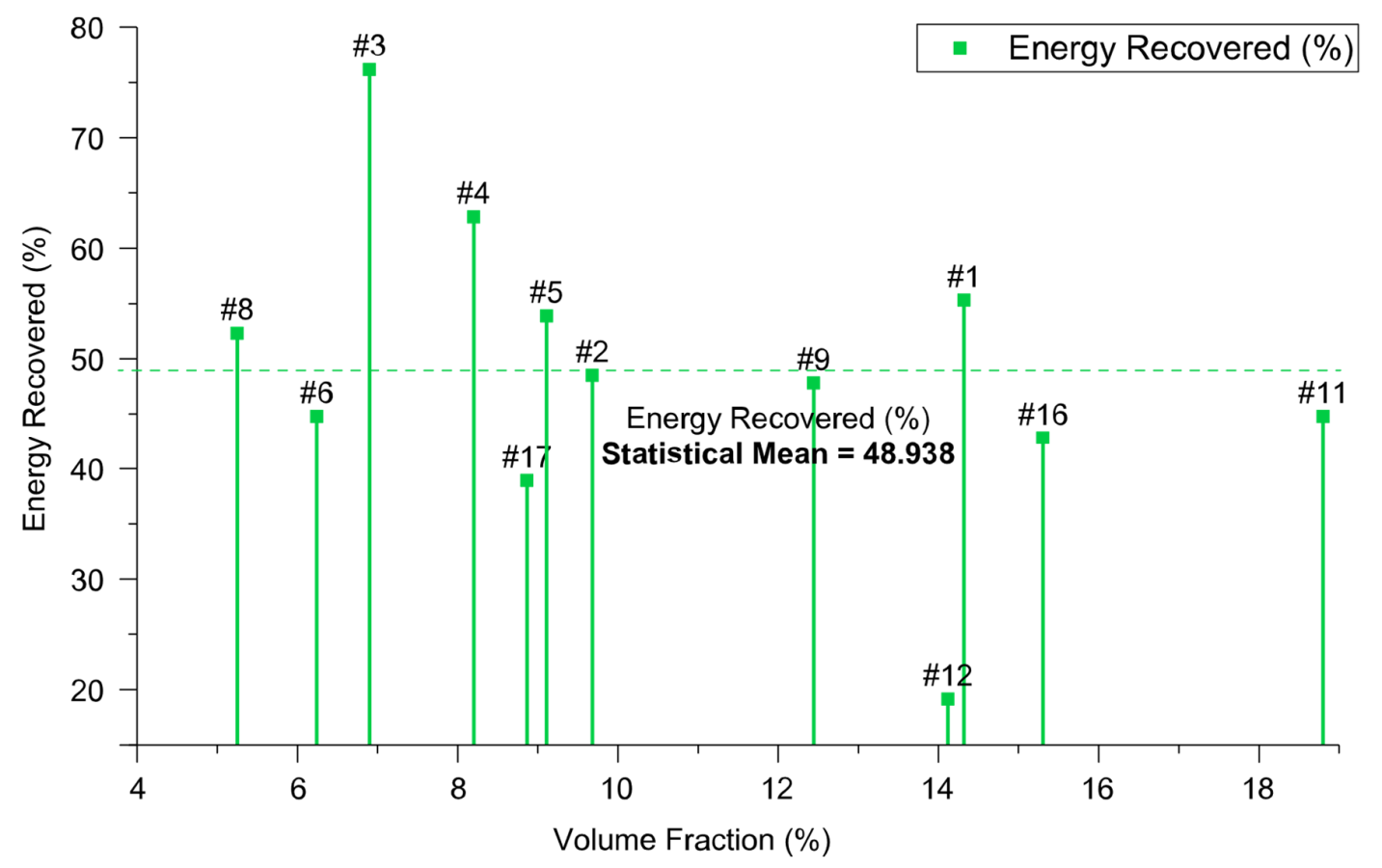

Figure 3. Overview of lattices' energy recovered

\section{Conclusion}

The here presented study describes the mechanical properties of lattice structures that consist of freeform unit cells. It starts by explaining the main drawbacks of conventional strut-based geometries, and how those shortcomings can be tackled with a different lattice design method by specifically employing industrial design competencies in CAD and research. In addition to optimizing the geometry alone, the generated freeform lattice variants exhibited a variety of behavioural modes. Our approach demonstrates how distinctive designs of unit cells influence the mechanical behaviour when combined into a lattice, hence resulting in either a rigid, stable structure or a compliant structure with elastic instability.

Positive anomalies identified against the overall trend in Figure 1 and Figure 2 demonstrate that volume fraction is not the main determinant for strength and stiffness properties, as well as strain and deformation mechanism. These mechanical properties are in fact greatly influenced by unit cell design and the freeform lattice's morphology. Among the 20 freeform lattices, design \#16 possessed above average values in both strength and yield strain while not experiencing drastic failure mode at $20 \%$ strain, which is unlike conventional materials where higher strength often results in brittleness. Well balanced designs as such could be further optimised for a potential application in high strength, compliant structures requiring large strain. It was also demonstrated that designs with characteristic ductility differ from those that exhibit drastic failure under $20 \%$ strain in terms of deformation behaviour. Designs that exhibited a return curve are generally observed to have either a homogenous deformation mode or slight barrelling, while those that showed drastic failure mode were observed to have simultaneous collapsing of top and bottom layers or layer-by-layer buckling. This seed experiment indicates that the unexplored possibilities of designing unit cells could offer a promising potential for future research in computer aided design and materials manufacturing.

A possible next direction would lie in the designing and combining of structurally compatible freeform lattices to create a heterogeneous, single part hierarchical lattice with both functionally stable and instable regions (Ion et al., 2016). These rationally designed heterogeneous lattices with amalgamated properties could lead to unprecedented outcomes with outstanding functional value (Lakes, 2002; Martin et al., 2015), for possible utilization in in load bearing, fluid exchange, heat transfer, acoustic, electromagnetic and photonic applications (Zok et al., 2016; Darabi, 2017; Sun and Li, 2018). 


\section{References}

Ahmadi, S.M., Yavari, S.A., Wauthle, R., Pouran, B., Schrooten J. et al. (2015), “Additively manufactured opencell porous biomaterials made from six different space-filling unit cells: The mechanical and morphological properties", Materials, Vol. 8 No. 4, pp. 1871-1896.

Anon (n.d.), Overview of materials for Nylon 12. [online] Available at: http://www.matweb.com/search/datasheetText.aspx?bassnum=O2640 (accessed 12.11.2017).

Anon (n.d.), Product information PA 2200 and PA 2201. [online] Available at: https://www.eos.info/pa-22002201-da42d3327dfc8bf2 (accessed 07.11.2017).

Anon (n.d.), P-Solutions Plastic laser sintering system FORMIGA P 110 for the direct manufacture of series, spare parts and functional prototypes. [online] Available at: https://cdn2.scrvt.com/eos/c9742dad885d8ac8/0a7acb2dca96/EOS_System_Data_Sheet_FORMIGA_P_100 EN_V2_Web.pdf (accessed 12.11.2017).

Ashby, M.F. (2005), Materials Selection in Mechanical Design, 3rd ed., Elsevier.

ASTM (2008), D695 Standard Test Method for Compressive Properties of Rigid Plastics, ASTM International, West Conshohocken, PA.

Bertoldi, K., Reis, P.M., Willshaw, S. and Mullin, T. (2010), "Negative poisson's ratio behavior induced by an elastic instability", Advanced Materials, Vol. 22 No. 3, pp. 361-366.

Bottlang, M., Doornink, J., Lujan, T.J., Fitzpatrick, D.C., Marsh, J.L. et al. (2010), "Effects of construct stiffness on healing of fractures stabilized with locking plates", Journal of Bone and Joint Surgery, Vol. 92 No. 2, pp. 12-22. https://doi.org/10.2106/JBJS.J.00780

Carneiro, V.H., Meireles, J. and Puga, H. (2013), “Auxetic materials - A review”, Materials Science-Poland, Vol. No. 4, pp. 561-571. https://doi.org/10.2478/s13536-013-0140-6

Cronskär, M. (2011), The use of additive manufacturing in the custom design of orthopedic implants, Licentiate thesis, Mid Sweden University.

Darabi, A.C. (2017), "Failure mechanisms of additively manufactured porous biomaterials : Effects of porosity and type of unit cell”, Journal of the Mechanical Behavior of Biomedical Materials, Vol. 50, pp. 180-191. http://doi.org/10.1016/j.jmbbm.2015.06.012

Deshpande, V.S., Ashby, M.F. and Fleck, N.A. (2001a), "Foam Topology Bending Versus Stretching Dominated Architectures", Acta Materialia, Vol. 49, pp. 1035-1040.

Deshpande, V.S., Fleck, N.A. and Ashby, M.F. (2001b), "Effective properties of the octet-truss lattice material", Journal of the Mechanics and Physics of Solids, Vol. 49 No. 8, pp. 1747-1769.

Dow, T.A. and Scattergood, R.O. (2003), "Mesoscale and microscale manufacturing processes: Challenges for materials, fabrication and metrology", Proceedings of the ASPE winter topical meeting, Vol. 28, pp. 14-19.

Fleck, N. (2004), "An overview of the mechanical properties of foams and periodic lattice materials", Cellular Metals and Polymers 2004, pp. 1-4.

Helou, M. and Kara, S. (2017), "Design, analysis and manufacturing of lattice structures: an overview", International Journal of Computer Integrated Manufacturing, Vol. 31 No. 3, pp. 243-261. https://doi.org/10.1080/0951192X.2017.1407456

Ion, A., Frohnhofen, J., Wall, L., Kovacs, R., Alistar, M. et al. (2016), “Metamaterial Mechanisms", Proceedings of the 29th Annual Symposium on User Interface Software and Technology - UIST '16, pp. 529-539.

Gibson, L.J. and Ashby, M.F. (1988), Cellular Solids: Structure And Properties, Pergamon Press, Oxford. https://doi.org/10.1002/crat.2170250912

Kolken, H.M.A. and Zadpoor, A.A. (2017), “Auxetic mechanical metamaterials”, RSC Advances, Vol. 7 No. 9 , pp. 5111-5129.

Lakes, R. (1993), "Materials with structural hierarchy", Nature, Vol. 361 No. 6412, pp. 511-515. https://doi.org/10.1038/361511a0

Lakes, R.S. (2002), "High damping composite materials: Effect of structural hierarchy", Journal of Composite Materials, Vol. 36 No. 3, pp. 287-297. https://doi.org/10.1177/0021998302036003538

Li, D., Dai, N., Zhou, X., Huang, R. and Liao, W. (2017), "Self-supporting interior structures modeling for buoyancy optimization of computational fabrication", International Journal of Advanced Manufacturing Technology, Vol. 95 No. 1-4, pp. 825-834. https://doi.org/10.1007/s00170-017-1261-6

Liu, F., Zhang, D., Zhang, P., Zhao, M. and Jafar, S. (2018), "Mechanical Properties of Optimized Diamond Lattice Structure for Bone Scaffolds Fabricated via Selective Laser Melting”, Materials, Vol. 11 No. 3, pp. 374. https://doi.org/10.3390/ma11030374

Martin, J.J., Fiore, B.E. and Erb, R.M. (2015), "Designing bioinspired composite reinforcement architectures via 3D magnetic printing”, Nature Communications, Vol. 6 No. 8641. https://doi.org/10.1038/ncomms9641

McCormack, J.P., Dorin, A. and Innocent, T.C. (2005), "Generative design: a paradigm for design research", Proceedings of Futureground, Design Research Society, Melbourne. 
Mir, M., Ali, M.N., Sami, J. and Ansari, U. (2014), "Review of mechanics and applications of auxetic structures", Advances in Materials Science and Engineering, Vol. 2014. http://doi.org/10.1155/2014/753496

Qiao, J.X. and Chen, C.Q. (2015), "Impact resistance of uniform and functionally graded auxetic double arrowhead honeycombs", International Journal of Impact Engineering, Vol. 83, pp. 47-58.

Qin, Z, Jung, G.S., Kang, M.J. and Buehler, M.J. (2017), "The mechanics and design of a lightweight threedimensional graphene assembly", Science Advances, Vol. 3 No. 1, pp. e1601536. https://doi.org/10.1126/sciadv.1601536

Sanami, M., Ravirala, N., Alderson, K. and Alderson, A. (2014), "Auxetic materials for sports applications", Procedia Engineering, Vol. 72, pp. 453-458.

Saunders, M. (2017), Lightweight lattices liberate new product performance. [online] Available at: http://www.altairenlighten.com/opinion/lightweight-lattices-liberate-new-product-performance/ (accessed 02.03.2018).

Shea, K, Aish, R. and Gourtovaia, M. (2005), "Towards integrated performance-driven generative design tools", Automation in Construction, Vol. 14 No. 2, pp. 253-264. https://doi.org/10.1016/j.autcon.2004.07.002

Sun, Y. and Li, Q.M. (2018), "Dynamic compressive behaviour of cellular materials: A review of phenomenon, mechanism and modelling”, International Journal of Impact Engineering, Vol. 112, pp. 74-115. https://doi.org/10.1016/j.ijimpeng.2017.10.006

Sundaram, M.M. and Ananthasuresh, G.K. (2009), "Gustave Eiffel and his optimal structures”, Resonance, Vol. 14 No. 9, pp. 849-865.

Vaezi, M., Seitz, H. and Yang, S. (2013), "A review on 3D micro-additive manufacturing technologies", International Journal of Advanced Manufacturing Technology, Vol. 67 No. 5-8, pp. 1721-1754.

Vigliotti, A. and Pasini, D. (2013), "Mechanical properties of hierarchical lattices", Mechanics of Materials, Vol. 62 , pp. 32-43.

Wang, X.-T., Li, X.-W. and Ma, L. (2016), "Interlocking assembled 3D auxetic cellular structures", Materials and Design, Vol. 99, pp. 467-476. https://doi.org/10.1016/j.matdes.2016.03.088

Wettergreen, M.A., Bucklen, B.S., Starly, B., Yuksel, E., Sun, W. and Liebschner, M.A.K. (2005), "Creation of a unit block library of architectures for use in assembled scaffold engineering", Computer-Aided Design, Vol. 37 No. 11, pp. 1141-1149. https://doi.org/10.1016/j.cad.2005.02.005

Zok, F.W., Latture, R.M. and Begley, M.R. (2016), "Periodic truss structures", Journal of the Mechanics and Physics of Solids, Vol. 96, pp. 184-203. https://doi.org/10.1016/j.jmps.2016.07.007

Zok, F.W., Latture, R.M. and Begley, M.R. (2016), "Periodic truss structures", Journal of the Mechanics and Physics of Solids, Vol. 96, pp. 184-203.

Axel Thallemer, Univ.-Prof. Dipl.-Ing.

National University of Singapore

4 Architecture Drive, 117566 Singapore, Singapore

Email: Axel.Thallemer@nus.edu.sg 